\title{
Smoothness of the velocity field and three-dimensional surface detection from optic flow
}

\author{
GEORGE J. ANDERSEN and PAUL ATCHLEY \\ University of Califormia, Riverside, California
}

\begin{abstract}
Five experiments were conducted to determine the importance of smoothness of the velocity field in detecting 3-D surfaces from optic flow. Subjects were presented with optic flow displays simulating either points positioned on a corrugated 3-D surface or points randomly positioned within a 3-D volume. The subject's task was to indicate whether or not the display appeared to be a 3-D surface. Smoothness of the velocity field was examined by systematically varying the speed of individual velocities in the flow field according to a Gaussian distribution with $M=0$ and $S D=\sigma$. Variations in frequency, amplitude, density, and surface complexity were also examined. Detection of the corrugated surfaces systematically declined with an increase in $\sigma$. An increase in frequency of the corrugation for simple (single-frequency corrugation) surfaces resulted in a decrease in surface detection accuracy. Accuracy increased with an increase in density and amplitude for both simple and complex (multiplefrequency corrugation) surfaces. An analysis of the deformation of the displays predicted performance on the basis of human observers, providing further support for the importance of deformation for 3-D surface detection.
\end{abstract}

Optic flow (Gibson, 1966; see also Helmholtz, 1867/ 1962) provides information useful for the recovery of 3-D shape. Previous research has demonstrated that optic flow can be used to recover surface slant and orientation (Braunstein \& Andersen, 1981; Braunstein \& Payne, 1969; Braunstein \& Tittle, 1988), recover the shape (Rogers \& Graham, 1979) and curvature of a surface (Norman \& Lappin, 1992; Todd, 1984), and detect the rigidity of surfaces (Braunstein, Hoffman, \& Pollick, 1990; De Bruyn \& Orban, 1990; Todd, 1984). In addition to empirical research on optic flow, there has been considerable interest in formal analyses of optic flow for the recovery of shape and depth information (Koenderink, 1986; Koenderink \& van Doorn, 1977; Longuet-Higgins \& Prazdny, 1980; Verri, Girosi, \& Torre, 1990). For example, Koenderink mathematically demonstrated that the divergence and deformation components of the flow field could be used for 3-D shape recovery.

An important assumption of this approach, as well as other approaches (e.g., Droulez \& Cornilleau-Pérès, 1990; Verri et al., 1990), is that the velocity field is locally smooth. Recent research on the detection of 3-D surfaces from motion has also suggested that smoothness of the velocity field is important for surface detection. Specifically, Andersen (1996) and Turner, Braunstein, and An-

This research was supported by National Science Foundation Grants BNS 9021081 and SBR 95-10431. The authors would like to thank Mike Braunstein for comments on an earlier draft of this manuscript. Correspondence should be addressed to G. J. Andersen, Department of Psychology, University of California, Riverside, CA 92521 (e-mail: andersen@) ucracl.ucr.edu). dersen (1995) have proposed analyses based on smoothness for the detection of 3-D surfaces from optic flow and structure from motion, respectively. Given that several analyses for 3-D surface detection and for 3-D shape recovery assume that the velocity field is smooth, an important issue is whether the detection and/or recovery of 3-D shape is altered when smoothness of the velocity field is violated.

Previous research has addressed this issue by examining 3-D shape recovery when the flow field contains multiple overlapping transparent surfaces that are separated in depth. During translation of this type of display, the velocities in the image vary from local region to local region. This variation of local velocity is the result of the depth separation of the transparent surfaces within the display. Results of studies by Andersen (1989; see also DeBruyn \& Orban, 1993) indicate that subjects can detect up to three transparent overlapping surfaces when the local smoothness of the velocity field is violated. Research by Andersen and Wuestefeld (1993) examined the detection of a 3-D surface positioned in front of and behind two transparent velocity fields. Their results suggest that the visual system segregates the overlapping velocity fields on the basis of underlying distributions of velocity present in the display. More recently, studies have assessed what statistical properties are used in this segregation process (Atchley \& Andersen, 1995) and assessed the ability of human observers to detect a gap between two overlapping velocity distributions (Turner \& Braunstein, 1994). The results of these studies, taken together, suggest that a segregation process occurs prior to analyses of optic flow that assume a smooth velocity field. 
It is important to note that the investigation of overlapping velocity fields represents one method of violating smoothness of the velocity field. A second method of violating smoothness is to systematically vary the smooth velocity field by adding noise to the velocity components that define a specific 3-D surface. This can be accomplished by randomly adding or subtracting a velocity component to the individual velocities of a display. A segregation process such as that proposed for overlapping transparent velocity fields (Andersen \& Wuestefeld, 1993; Atchley \& Andersen, 1995) would be ineffective in analyzing smoothness violations produced by this method.

The purpose of the present study was to examine the ability of human observers to detect surfaces from optic flow when smoothness was violated in this manner. The present study examined this issue by presenting subjects with displays in which points that define a surface were systematically perturbed off the surface. Subjects were presented with flow fields that defined either a corrugated surface or a random pattern. The smoothness of the velocity field was varied by systematically adding or subtracting a horizontal velocity component, henceforth referred to as "velocity noise," from a Gaussian distribution with mean of zero and variance of $\sigma$. This resulted in velocity components that could be either positive or negative and thus would be either added to or subtracted from the original velocities defined by the corrugated surface. An increase in $\sigma$ would result in an increase in the overall magnitude of the noise velocities, in turn resulting in a greater perturbation in the velocities that define the corrugated surface. This perturbation in local velocities of the display is consistent with systematically perturbing the points off the surface (see Figure 1) and increasing the nonsmoothness (local variation of surface orientation) of the simulated surface.

In the present study, we manipulated the magnitude of nonsmoothness by systematically varying $\sigma$. This method allowed us to vary the overall magnitude of the non-
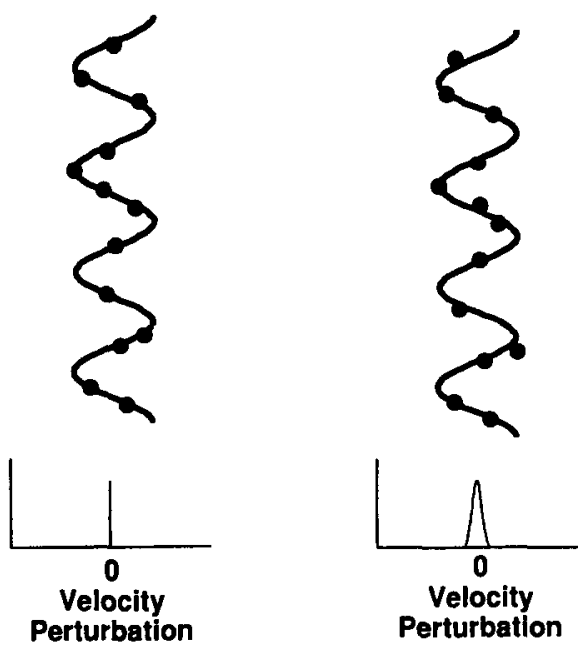

smoothness of the velocity field. In Experiments 1-3, we employed a yes/no detection paradigm without feedback, similar to that used in previous studies on surface detection from motion (Andersen, 1996; Andersen \& Wuestefeld, 1993; Turner et al., 1995). In Experiments 1 and 2, the importance of density, amplitude, and corrugation frequency was examined for the detection of single sine wave corrugation surfaces. In Experiment 3, we examined the importance of density and amplitude in the detection of complex surfaces based on sums of sine frequencies. Experiments 4 and 5 examined the importance of density, amplitude, and corrugation frequency using feedback and a two-alternative forced-choice (2AFC) paradigm. In addition, we conducted simulations of the deformation analysis proposed by Andersen (1996) to assess the tolerance of this analysis to systematic variations in local smoothness. The results of these simulations were compared with the performance of human observers.

\section{EXPERIMENT 1}

The purpose of the first experiment was to examine the detection of simple (single-frequency) corrugated surfaces from optic flow when smoothness of the velocity field was varied. Subjects were presented with displays simulating either a 3-D corrugated surface or a random volume (Figure 2). Smoothness was varied in the following manner. The velocity of each point was altered by an amount sampled from a Gaussian distribution of speeds with a mean of 0 and a standard deviation of $\sigma$. In order to assess the minimal density needed to detect the presence of a surface at each $\sigma$, the number of points in the display was varied. In addition, surface structure was examined by varying the spatial frequency of the corrugated pattern.

\section{Method}

Subjects. The subjects were 5 students at the University of California, Riverside, who were paid for their participation. Four of the

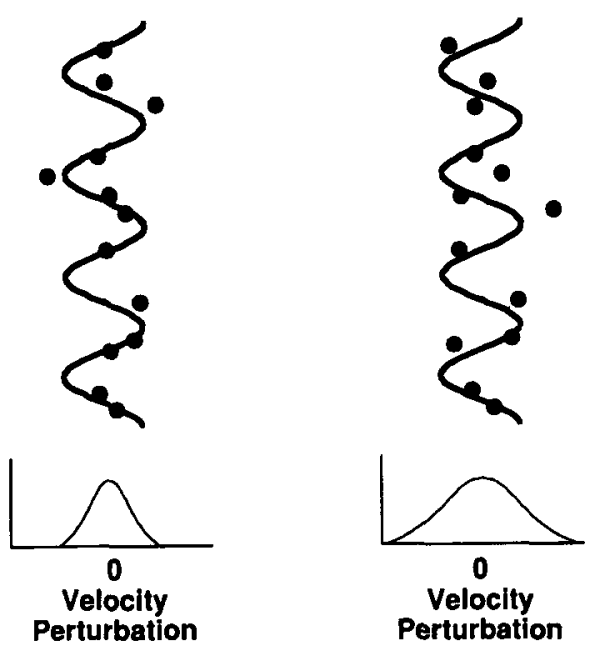

Figure 1. Schematic diagram of a side view of a corrugated surface. The graphs at the bottom of each illustration are Gaussian distributions of velocity noise with $M=0$. From left to right are increased standard deviations of the noise distribution. An increase in the standard deviation of noise is consistent with systematically perturbing the points off the surface as is illustrated in the corrugated surfaces from left to right. 


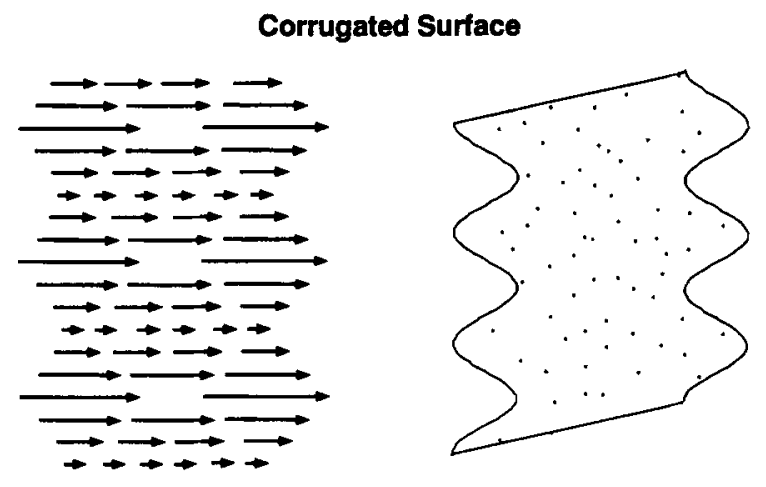

Random Volume
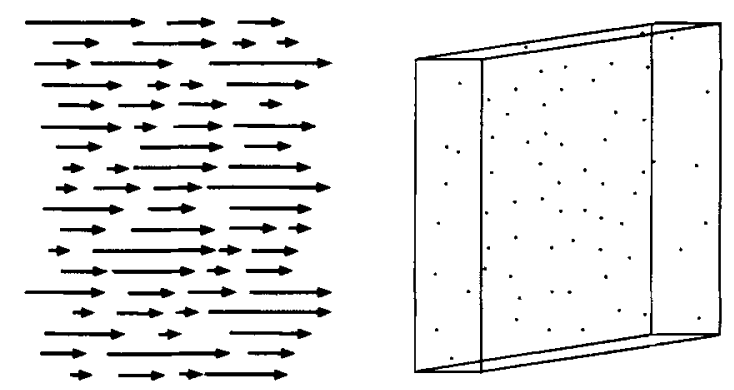

Figure 2. Examples of two optic flow fields examined in the present research. The top flow field is the velocity field produced by perspective horizontal translation of a 3-D surface corrugated in depth according to a sine function. The bottom flow field is a randomized version, with the velocities repositioned randomly in the image. The velocity vectors are regularly spaced along the vertical axis for purposes of illustration. In the present experiments, the velocities were randomly positioned in the image.

subjects were naive with regard to the purpose of the experiment. All subjects had normal or corrected-to-normal vision.

Design. Three independent variables were examined: spatial frequency of the corrugated surface $(0.34,0.57,0.75,0.94$, and 1.25 cycles/deg of visual angle), texture density $(2.61,7.2$, and 11.78 dots $\left./ \operatorname{deg}^{2}\right)$, and the standard deviation of Gaussian velocity noise $(0, .22, .45, .67, .9$, or $1.12 \mathrm{deg} / \mathrm{sec})$. Subjects were presented with 30 surface displays for each condition and an equal number of matched random displays.

Stimuli. The stimuli were computer-generated displays that simulated points positioned either on a 3-D surface or randomly within a volume (Figure 2 ). The surface displays were generated in the following manner: Points were randomly positioned in the image, and a horizontal translation velocity was assigned to each point according to a sine function of vertical position. Specifically, the position change of a point in the image per frame $i$ was determined using the following equation:

$$
i=a \sin \left(f 2 \pi y / y_{\max }\right)+a+b,
$$

where $a$ is the amplitude of the corrugated surface, $b$ is the minimum horizontal motion per frame, $f$ is the frequency of the corrugated surface, $y$ is the vertical coordinate (in pixel units) of the point, and $y_{\max }$ is the maximum $y$ value. This simulated a sinusoidal surface in depth that was corrugated in the vertical dimension. To produce the random volume conditions, the points and velocities of the corrugated surface displays were randomly reassigned to different vertical positions in the image (Figure 2). This resulted in a velocity field in which the velocities varied randomly along the ver- tical axis. These two display conditions have identical distributions of velocities in the image. However, in the random condition, the velocities did not vary systematically with the vertical position. Velocities for both the surface and random conditions ranged between $1.95 \mathrm{deg} / \mathrm{sec}$ and $2.84 \mathrm{deg} / \mathrm{sec}$. The random displays thus simulated points randomly distributed in a volume having the same overall depth as the surface displays.

The local smoothness of the surface was systematically varied by perturbing the velocity of points, and hence the position of points on the surface, in the following manner. Perturbations were randomly selected from a Gaussian distribution of mean $=0$ and $S D=$ $\sigma$. The magnitude of the perturbation was determined by the parameter $\sigma$. For $\sigma=0$, there would be no perturbations. For $\sigma>0$, a positive or negative horizontal velocity component would be added to the velocity of each point that defined the surface. The perturbations for each point were constant throughout a display sequence. The perturbed displays represent rigid motion of points that were in front of or behind the smooth surface by varying amounts.

The displays subtended an $8.74^{\circ}$ square region. The size of the dots were $0.51 \mathrm{~min}$ arc. Whenever a dot was projected outside the image area during the horizontal translation, it was relocated at the opposite horizontal position in the image at the same $y$-coordinate. The display was updated at $27 \mathrm{~Hz}$. The displays oscillated once (motion left to right and right to left) over a 96-frame cycle. The display duration was 3.55 sec.

Apparatus. The displays were generated on an Evans and Sutherland ESV30 graphics workstation (resolution $1,280 \times 1,024$ ). Subjects viewed the displays through a viewing tube that restricted the field of view to a circular area $8.7^{\circ}$ in diameter. A series of corrugated surface models of different frequencies and amplitudes were used to illustrate the types of surfaces subjects would be asked to detect. The models were constructed of sheet metal and painted black with white dots affixed to the models.

Procedure. The subjects participated individually. They were instructed that they would be viewing a series of displays of moving white dots and that on some trials the dots would appear to form a corrugated 3-D surface. Subjects were shown the corrugated surface models as examples of the types of surfaces that were likely to be presented. On other trials, the dots would appear as a random 3-D pattern. Subjects were instructed that their task was to determine whether the moving points appeared to form a corrugated surface in depth. Subjects used the numeric keypad on the keyboard to respond. Subjects were asked to respond at the end of each display by pressing a " 1 " if they perceived a $3-D$ surface or a " 0 " if they did not perceive a 3-D surface.

Subjects viewed the displays monocularly in a dark room at a distance of $1.7 \mathrm{~m}$. They participated in three 60 -min sessions run on separate days. Each session consisted of 10 blocks. Each block consisted of a single presentation of each of the 180 displays in random order. Thus, each subject viewed 30 trials of each display condition for a total of 5,400 trials. Subjects at the beginning of the first session were shown the five surfaces at the highest density level and were told that these were the surfaces they would be asked to detect during the experiment. An additional block of trials was run at the beginning of each session and was used as practice. The practice trials included displays with density levels that in previous research (Andersen, 1996; Andersen \& Wuestefeld, 1993) resulted in 100\% accuracy in detecting a surface. No feedback was provided to the subjects during either the practice or the test trials. All subjects during the practice trials correctly detected the corrugated surfaces with values of $\sigma=0$. At the end of the experiment, subjects were asked about any strategy they had used to respond to the displays.

\section{Results and Discussion}

All subjects reported a compelling perception of depth from the displays, with some of the displays producing a compelling impression of a 3-D surface. $\mathrm{A} d^{\prime}$ was com- 
puted (the hit rate was the proportion of surface display trials on which subjects indicated a perception of a surface; the false alarm rate was the proportion of random display trials on which subjects indicated a perception of a surface) for each subject for each surface frequency, density, and velocity noise level (Green \& Swets, 1966). A within-subjects three-way (velocity noise $X$ frequency $X$ density) analysis of variance (ANOVA) was conducted using the $d$ 's as the dependent variable.

The main effect of corrugation frequency was significant $[F(4,16)=10.12, p<.01]$. Mean sensitivity in detecting the $0.34,0.57,0.75,0.94$, and 1.25 cycles/deg corrugation surfaces were $2.19,2.5,2.1,1.78$, and 1.27, respectively. Post hoc comparisons (Tukey's HSD test) showed significant differences between the 0.94 cycles/ deg and the $0.34,0.57$, and 0.75 cycles/deg corrugation frequencies. In addition, significant differences were found between the 1.25 cycles/deg and the $0.34,0.57$, and 0.75 cycles/deg corrugation frequencies. These results indicate that an increase in corrugation frequency resulted in a decrease in detection performance, a result previously reported in studies examining surface detection in the presence of separate noise points (Andersen \& Wuestefeld, 1993) and surface detection without noise (Andersen, 1996).

The main effect of density was significant $[F(2,8)=$ 84.2, $p<.01]$. Mean sensitivities for the 2.61, 7.2, and $11.2 \mathrm{dots} / \mathrm{deg}^{2}$ conditions were $1.21,2.25$, and 2.47 , respectively. Post hoc comparisons showed significant differences between the $2.61 \mathrm{dots} / \mathrm{deg}^{2}$ condition and the 7.2 and $11.2 \mathrm{dots} / \mathrm{deg}^{2}$ conditions.

The main effect of velocity noise was significant $[F(5,16)=10.12, p<.01]$. Mean sensitivities for the 0 , $0.22,0.45,0.67,0.9$, and $1.12 \mathrm{deg} / \mathrm{sec}$ conditions were $3.26,3.12,2.74,1.52,0.94$, and 0.35 , respectively. Post hoc comparisons showed mean $d$ 's to be significantly higher for the 0.0 variance condition compared with all other variance levels. The mean $d^{\prime}$ for the $0.22 S D$ velocity noise condition was significantly greater than the mean $d^{\prime}$ 's for the $0.67,0.9$, and $1.12 S D$ velocity noise conditions. The mean $d^{\prime}$ for the 0.45 velocity noise condition was significantly greater than the mean $d^{\prime} \mathrm{s} 0.67,0.9$, and 1.12 velocity noise conditions. Finally, the mean $d^{\prime}$ for the 0.67 velocity noise condition was significantly greater than the mean $d^{\prime}$ for the 1.12 condition.

The two-way interaction between velocity noise and density was significant $[F(10,40)=3.39 p<.01]$. This interaction is shown in Figure 3. As is shown in Figure 3, there was little effect of low levels of velocity noise $(0.22$ and $0.45 \mathrm{deg} / \mathrm{sec}$ conditions) for the highest density levels examined. However, sensitivity decreased at these levels of velocity noise with the lowest density level examined. These results, taken together with the main effect of velocity noise, indicate that the visual system can tolerate perturbations in the velocities of points in the display. These results also indicate that performance gracefully degraded with increasing noise, suggesting that there is no specific noise velocity value in which performance, across all conditions, drops to chance. In addition, the

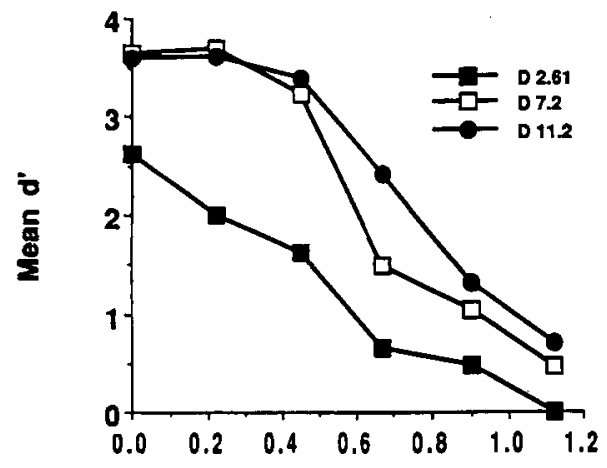

S. D. of Velocity Noise (deg/sec)

Figure 3. Mean $d^{\prime}$ as a function of velocity noise and density from Experiment 1.

two-way interaction of velocity noise and density indicates a greater effect of velocity noise on surface detection for low-density than for high-density conditions.

The two-way interaction between corrugation frequency and density was significant $[F(8,32)=6.57, p<$ $.01]$. As is shown in Figure 4, sensitivity decreased more rapidly for the 2.6 density condition than for the 7.2 and 11.2 density conditions. There were no other significant interactions.

\section{EXPERIMENT 2}

In the first experiment, the surfaces were defined as a repeating corrugated pattern based on a sine wave function. Previous research (Andersen, 1996; Andersen \& Wuestefeld, 1993) has indicated that the detection of corrugated surfaces is affected by the amplitude of the corrugated surface, with increased detection performance for high-amplitude than for low-amplitude corrugated surfaces. The purpose of the second experiment was to examine the effects of amplitude on surface detection when the smoothness of the flow field was systematically perturbed. In addition, Experiment 2 examined a limited set of frequency and density conditions.

\section{Method}

Subjects. The subjects were 4 graduate students and 1 undergraduate student at the University of California, Riverside, who were paid for their participation. All were naive with regard to the purpose of the experiment. Acuity of at least 20/40 (Snellen Eye Chart) was required in the eye used in the experiment.

Design. Four independent variables were examined: texture density ( 2.6 or $11.0 \mathrm{dots} / \mathrm{deg}^{2}$ ), depth range of the display (maximum to minimum velocity ratio of $1.5,2.0$, or 2.5 ), corrugation frequency $(0.58$ or 1.16 cycles $/ \mathrm{deg})$ and the standard deviation of Gaussian noise $(0,0.22,0.45,0.67,0.9$, or $1.12 \mathrm{deg} / \mathrm{sec})$. Subjects were presented with 30 surface displays for each condition and an equal number of random displays.

Stimuli. The stimuli were similar to those used in the first experiment with the following exception. The maximum and minimum velocities of the displays were $1.62 \mathrm{deg} / \mathrm{sec}$ and $1.08 \mathrm{deg} / \mathrm{sec}$ (for the 1.5 velocity ratio displays), $1.8 \mathrm{deg} / \mathrm{sec}$ and $0.9 \mathrm{deg} / \mathrm{sec}$ (for the 2.0 velocity ratio displays), and $1.92 \mathrm{deg} / \mathrm{sec}$ and $.77 \mathrm{deg} / \mathrm{sec}$ (for the 2.5 velocity ratio displays). 


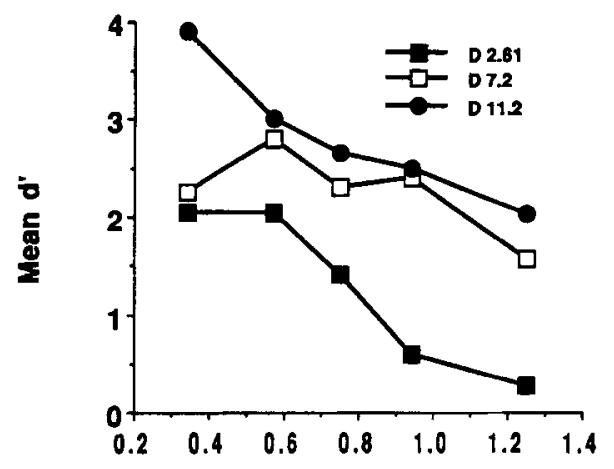

Corrugation Frequency (cycles/deg)

Figure 4. Mean $d^{\prime}$ as a function of corrugation frequency and density from Experiment 1.

Apparatus. The same apparatus was used as in Experiment 1. Procedure. The procedure was the same as Experiment 1 with the following exceptions. Subjects were shown prior to the first session the four different surfaces they were to detect during the experiment. Subjects participated in ten 1 -h sessions run on separate days. Each session consisted of three blocks of each display presented in a random order. Thus, each subject viewed 30 trials of each display condition for a total of 4,320 trials. No feedback was provided to the subjects at any time during the experiment.

\section{Results and Discussion}

A $d^{\prime}$ was computed (the hit rate was the proportion of surface display trials on which subjects indicated a perception of a 3-D surface; the false alarm rate was the proportion of random display trials on which subjects indicated a perception of a 3-D surface) for each subject for each surface frequency, amplitude, velocity noise, and density level (Green \& Swets, 1966). A withinsubjects four-way (velocity noise $\times$ frequency $\times$ density $X$ amplitude) ANOVA was conducted using the $d^{\prime}$ 's as the dependent variable. The results of Experiment 2 are shown in Figure 5. The main effect of corrugation frequency was significant $[F(1,4)=45.1, p<.01]$. This result indicates that greater sensitivity was observed for detecting the 0.58 cycles/deg surface (average $d^{\prime}$ of 3.23 ) than the 1.16 cycles/deg surface (average $d^{\prime}$ of 2.66 ). Greater sensitivity was also observed for the high- (average $d^{\prime}$ of 3.58) than for the low- (average $d^{\prime}$ of 2.31) density condition $[F(1,4)=579.1, p<.01]$. In addition, the main effect of depth range was significant $[F(2,8)=$ $17.1, p<.01]$. The average $d$ 's for the $1.5,2.0$, and 2.5 velocity ratios were $2.47,2.91$, and 3.48 , respectively. Post hoc comparisons indicated significant differences among all three depth ranges examined. These three main effects replicate the results of previous research ex-

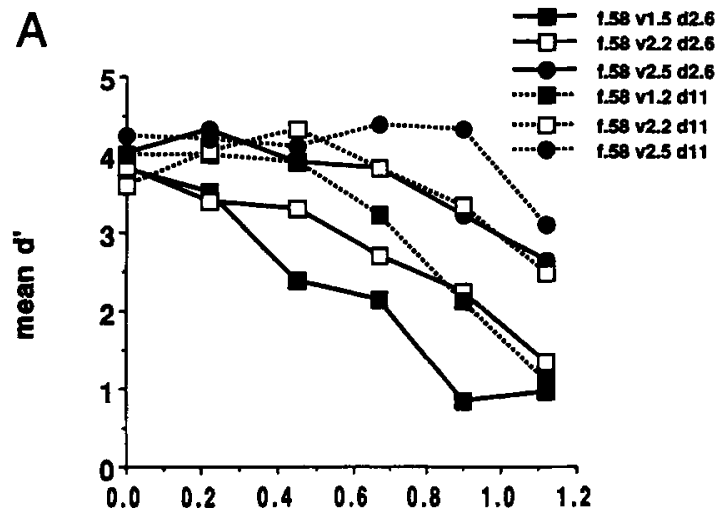

S. D. of Velocity Noise (deg/sec)

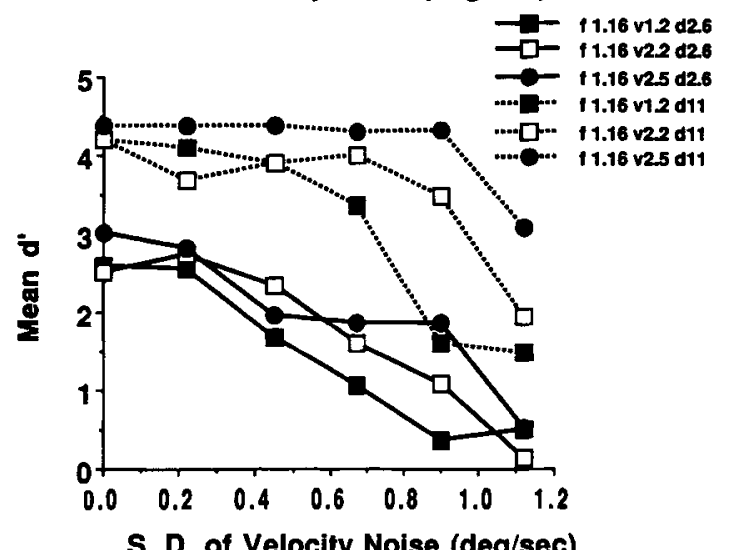

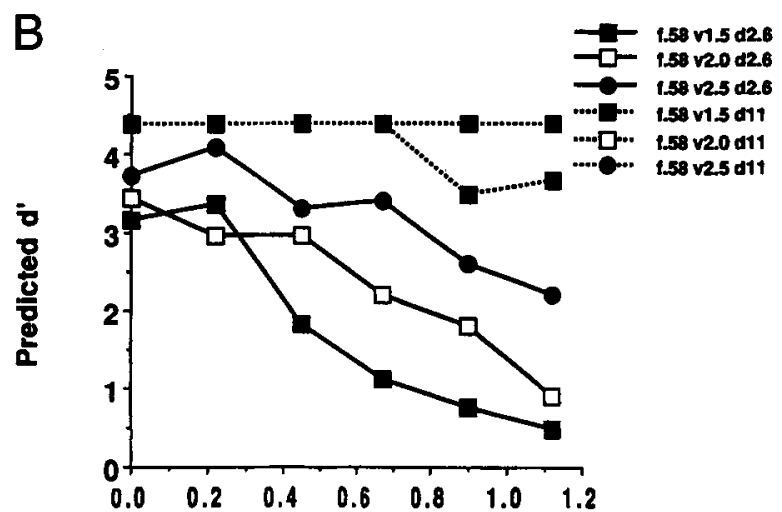

S. D. $($ deg/sec)

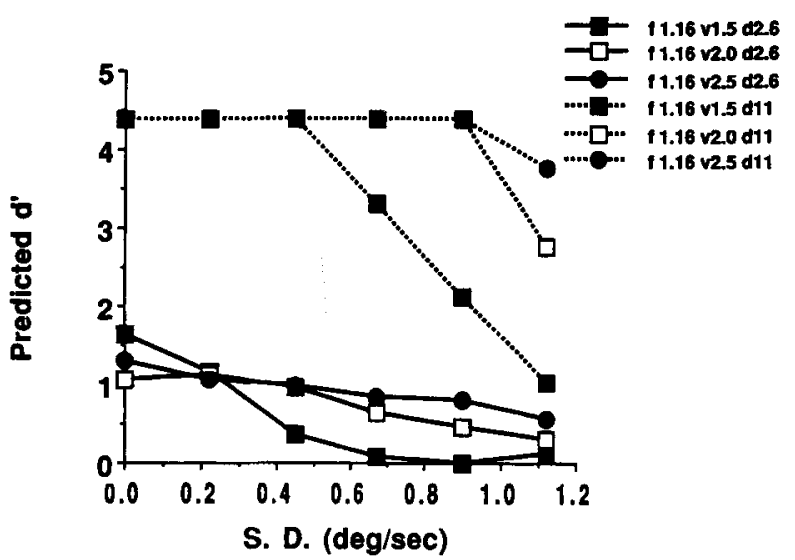

Figure 5. (A) Mean $d^{\prime}$ (see General Discussion) as a function of frequency (f), amplitude (v), density (d), and velocity noise from Experiment 2. (B) Predicted $d^{\prime}$ (see General Discussion) as a function of frequency (f), amplitude (v), density (d), and velocity noise as from Experiment 2. 
amining the detection of 3-D corrugated surfaces without noise (Andersen, 1996).

The main effect of velocity noise was significant $[F(5,20)=15.3, p<.01]$. The average $d^{\prime}$ 's for the $0,0.22$, $0.45,0.67,0.9$, and 1.12 velocity noise conditions were $3.71,3.63,3.35,3.04,2.39$, and 1.61 , respectively. These results indicate that detection performance systematically decreased with an increase in the perturbation of the smooth velocity fields that defined the corrugated surfaces.

The two-way interaction between amplitude and velocity noise was significant $[F(10,40)=4.69, p<.01]$ and is depicted in Figure 6. As shown in Figure 6, detection performance decreased systematically with an increase in velocity noise for all three amplitudes examined, with a steeper decrease in performance occurring with the lowest amplitude level examined. For example, the mean $d^{\prime}$ for the .9 velocity noise condition and the 1.5 velocity ratio condition was 1.18 . In contrast, the mean $d^{\prime}$ for the .9 velocity noise condition and the 2.5 velocity ratio condition was 3.52 . This result indicates that subjects were more tolerant of velocity noise for highamplitude than for low-amplitude conditions.

The interaction between density and velocity noise was also significant $[F(5,20)=5.1, p<.01]$ and is shown in Figure 7. This result indicates that sensitivity decreased, with increased levels of velocity noise, at a greater rate for low- than for high-density levels.

The results of the second experiment indicate that the effect of increased velocity noise on sensitivity varied according to the simulated amplitude of the surface. This result provides further support that there is no specific noise velocity value in which performance, across all conditions, drops to chance. In addition, the results of the second experiment confirm the finding in Experiment 1 that systematic variations in the smoothness of the velocity field resulted in a graceful degradation in detection performance.

\section{EXPERIMENT 3}

In the first experiment, the surfaces were defined as a repeating corrugated pattern based on a sine wave func-

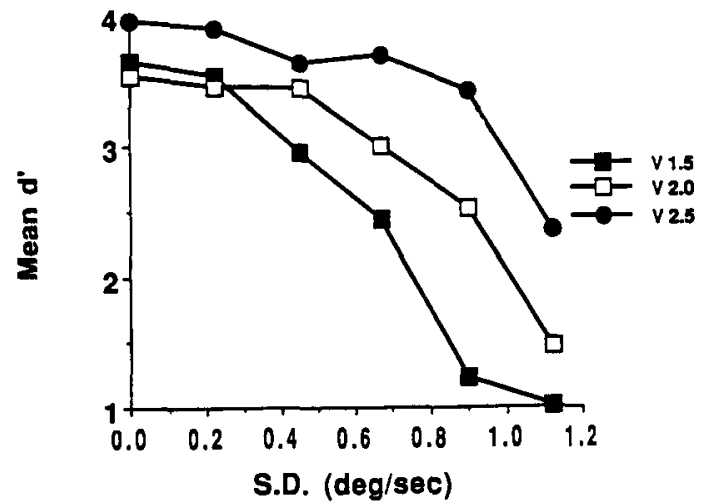

Figure 6. Mean $d^{\prime}$ as a function of amplitude (V) and velocity noise from Experiment 2.

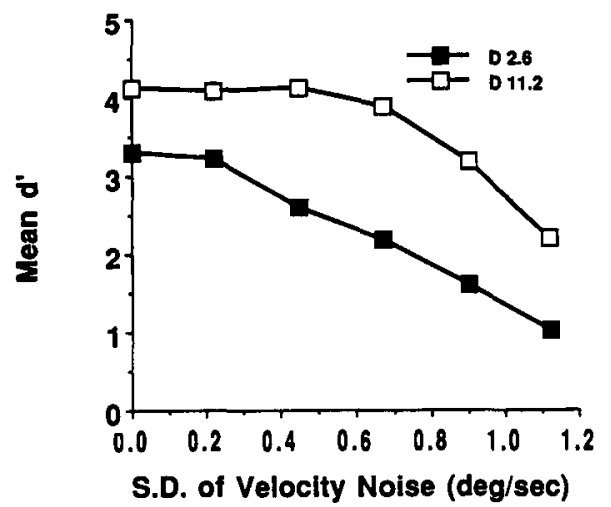

Figure 7. Mean $d^{\prime}$ as a function of density (D) and velocity noise from Experiment 2.

tion. Previous research (Andersen, 1996) indicated that the detection of surfaces under minimal density conditions varied according to whether the surface was a single sine wave corrugation or a complex corrugation surface. One explanation for the different results for simple and complex corrugation surfaces is that the presence of a repeating pattern for single sine wave surfaces may have provided a cue for detecting the surface. The purpose of the third experiment was to determine whether the effects of velocity noise in detecting single sine wave corrugated surfaces would be similar to the effects of noise in detecting complex sine wave corrugated surfaces.

In Experiment 3, we examined surfaces with corrugations produced by combining pairs of three $(3.78,1.89$, or $1.26 \mathrm{cycles} / \mathrm{deg}$ ) frequencies comparable to the complex corrugated surfaces examined in our previous research (Andersen, 1996). These frequencies were selected on the basis of two factors. First, two of the three frequencies used had resulted in high accuracy in our previous research. Second, combinations were selected that would generate nonrepeating patterns that were distinct from each other. The results of our previous experiments showed no significant difference in detection performance, according to the type of complex corrugation, across variations of amplitude and density. In the present experiment, we examined a fourth corrugated surface, defined by the linear combination of all three frequencies.

In addition, the amplitude of the corrugated surfaces was varied to assess whether the same pattern of results obtained using single sine wave surfaces would occur for irregular smooth surfaces. One problem in using sums of sine wave corrugations to define a complex corrugated surface is that the overall range of velocities will vary based on the single sine wave corrugations used to define the surface. To ensure that the range of velocities, for a specific amplitude level, were equivalent for each corrugation pattern, the sums of the frequencies were scaled.

\section{Method}

Subjects. The subjects were 3 graduate students and 1 undergraduate student at the University of California, Riverside, who were paid for their participation. All were naive with regard to the 
purpose of the experiment. Acuity of at least 20/40 (Snellen Eye Chart) was required in the eye used in the experiment.

Design. Four independent variables were examined: texture density (2.6, or $\left.11.2 \mathrm{dots} / \mathrm{deg}^{2}\right)$, depth range of the display (maximum to minimum velocity ratio of 1.5 or 2.5 ), surface type (one of four simulated complex surfaces), and the standard deviation of velocity noise $(0,0.22,0.45,0.67,0.9$, or $1.12 \mathrm{deg} / \mathrm{sec})$. Subjects were presented with 30 surface displays for each condition and an equal number of matched random displays.

Stimuli. The stimuli were the same as those used in the previous experiments with the following exception. The surfaces were defined by one of three complex sinusoids according to the following scheme. Three different sine waves $(3.78,1.89$, and 1.26 cycles/ $\mathrm{deg}$ ) were used to generate the displays. For three of the corrugated surfaces, image velocities (horizontal position per frame) defined according to the complex sinusoidal functions were generated by linear summation of a subset of the three frequencies using the following equation:

$$
i=s\left[a \sin \left(f_{1} 2 \pi y / y_{\max }\right)+\sin \left(a f_{2} 2 \pi y / y_{\max }\right)\right]+a+b,
$$

where $s$ is the scale factor, $a$ is the amplitude of the sine wave surfaces, $f_{1}$ and $f_{2}$ are the two frequencies used to define the complex surface, $y$ is the vertical position (in pixel units), $y_{\max }$ is the maximum vertical position (in pixel units), and $b$ is the minimum horizontal motion. Surfaces S1, S2, and S3 were generated by combining frequencies of 3.78 and $1.89,1.89$ and $1.26,3.78$ and 1.26 cycles/ deg. Surface $\$ 4$ was defined using the following equation:

$$
\begin{aligned}
i= & s\left[a \sin \left(f_{1} 2 \pi y / y_{\max }\right)+a \sin \left(f_{2} 2 \pi y / y_{\max }\right)\right. \\
& \left.+a \sin \left(f_{3} \pi y / y_{\max }\right)\right]+a+b,
\end{aligned}
$$

and incorporated all three frequencies of $1.26,1.89$, and $3.78 \mathrm{cy}-$ cles/deg. Linear combinations of these frequencies resulted in different amplitudes. In order to produce equivalent amplitudes across frequency pairs, mathematical simulations of the velocities used to define the surfaces were conducted to determine an appropriate scale factor for the amplitudes. For the 1.75 velocity ratio amplitudes, the scale factor for Displays 1, 2, 3, and 4 were 1.705, 1.575, 1.95 , and 1.37 , respectively. For the 3.4 velocity ratio amplitudes, the scale factor for Displays $1,2,3$, and 4 were $3.41,3.15,3.9$, and 2.74 , respectively.

Apparatus. The same apparatus was used as in Experiments 1 and 2 .

Procedure. The procedure was the same as Experiments 1 and 2 with the following exception. Subjects were shown the eight different surfaces at the highest density level prior to the practice trials.

\section{Results and Discussion}

A $d^{\prime}$ was computed (the hit rate was the proportion of surface trials on which subjects indicated a perception of a surface; the false alarm rate was the proportion of random display trials on which subjects indicated a perception of a 3-D surface) for each subject for each matched surface and random display. A within-subjects four-way (velocity noise $\times$ surface type $\times$ density $\times$ amplitude) ANOVA was conducted using the $d$ 's as the dependent variable. The main effect of surface type was significant $[F(3,12)=7.7, p<.01]$. Average $d^{\prime}$ s for the surface types $1,2,3$, and 4 were $1.18,1.51,1.79$, and .94 , respectively. Post hoc comparisons indicated significant differences between Surface 2 and Surfaces 3 and 4; and Surface 3 and Surfaces 1 and 4 . This result was surprising as our previous research (Andersen, 1996) had not found an effect of surface type for complex corrugated surfaces. There was also a significant two-way interaction between surface type and velocity noise $[F(15,60)=3.27, p<.01]$. The results of this interaction are shown in Figure 8. According to this result, differences between the different surface types increased with an increase in the standard deviation of velocity noise. Interestingly, a planned comparison for the zero noise condition indicated that no significant difference existed between the four surface types. This finding is consistent with the results of our previous research examining complex corrugated surfaces (Andersen, 1996). This finding, taken together with the main effect of surface type, suggest that the significant differences found for surface type are the result of significant differences between different surface types when the smoothness of the velocity fields defining each surface was perturbed.

The three-way interaction between amplitude, velocity noise, and surface type was also significant $[F(15,60)=$ $2.97, p<.01]$ and is shown in Figure 9. This interaction indicates that detection performance dropped more rapidly for the low-amplitude than for the high-amplitude displays. This conclusion is further supported by the significant three-way interaction between density, amplitude, and noise $[F(5,20)=3.81, p<.025]$, which is depicted in Figure 10. Finally, the main effect of amplitude was significant $[F(1,4)=27.1, p<.01]$. This result indicates that detection performance was greater for the high-amplitude (mean $d^{\prime}$ of 1.73) than for the lowamplitude displays (mean $d^{\prime}$ of .93 ).

Consistent with the results from Experiments 1 and 2, detection performance decreased with an increase in the magnitude of the noise $[F(5,20)=20.48, p<.01]$. The mean $d^{\prime}$ 's for the $0,0.22,0.45,0.67,0.9$, and 1.12 velocity noise conditions were $3.09,2.37,1.44,0.62,0.32$, and 0.31 , respectively. These results indicate that performance systematically degraded with an increase in velocity noise. The two-way interaction between noise and amplitude was significant $[F(5,20)=6.8, p<.01]$. This interaction is shown in Figure 11. This interaction indicates that performance decreased more rapidly, with increasing noise, for the low- than for the high-amplitude displays. The two-way interaction between density and noise was also significant $[F(5,20)=6.69, p<.01]$ and

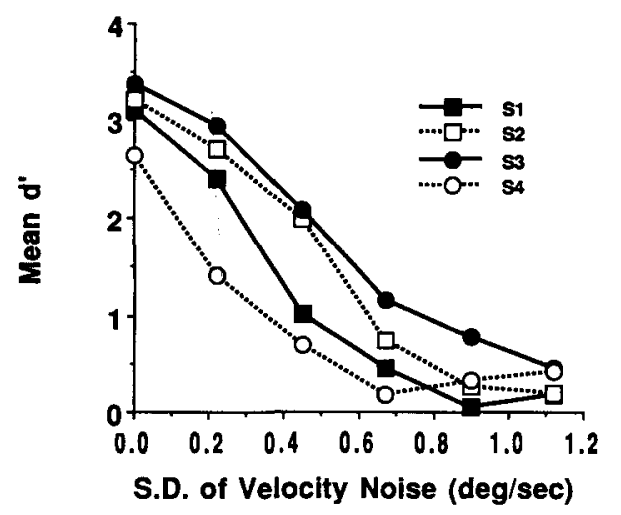

Figure 8. Mean $d^{\prime}$ as a function of surface type (S1, S2, S3, and S4) velocity noise from Experiment 3. 

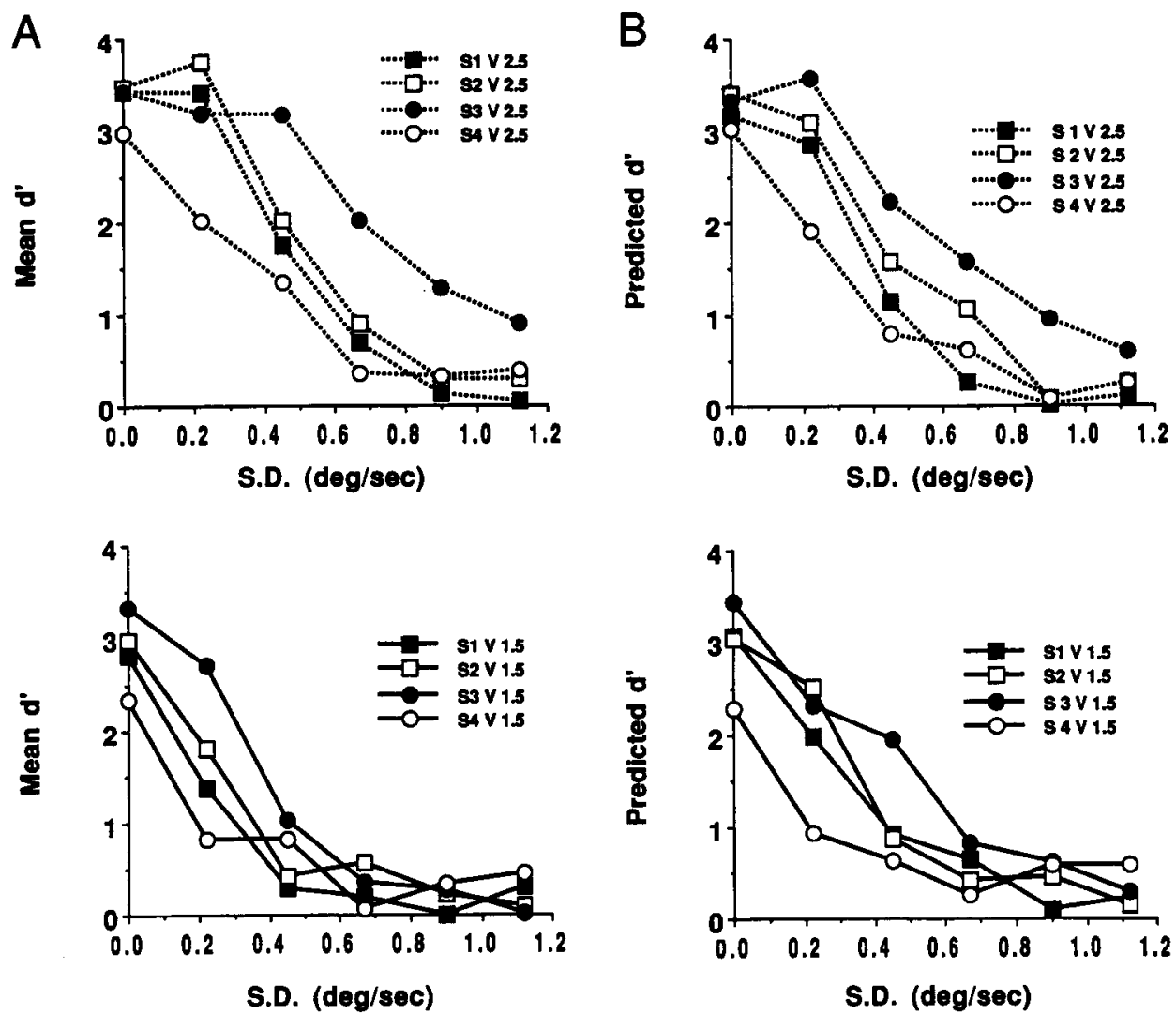

Figure 9. (A) Mean $d^{\prime}$ (see General Discussion) as a function of surface type (S), amplitude (V), and velocity noise from Experiment 3. (B) Predicted $d^{\prime}$ (see General Discussion) as a function of surface type (S), amplitude (V), and velocity noise from Experiment 3.

is shown in Figure 12. This result indicates that detection performance decreased more rapidly for the low- than for the high-density condition.

\section{EXPERIMENT 4: CONTROL STUDY}

In Experiments 1, 2, and 3, subjects were asked to detect displays that simulated a corrugated 3-D surface. These experiments used a yes/no detection paradigm in which subjects were presented with a single display and were then asked to indicate for each display whether the display appeared as a surface or random volume. In addition, no feedback was used. One potential concern regarding this paradigm is that a response bias by subjects might have varied from trial to trial. In addition, because feedback was not used, subjects may not have performed optimally. We therefore conducted two additional experiments in which a $2 \mathrm{AFC}$ paradigm was used with feedback. One advantage of the $2 \mathrm{AFC}$ paradigm is that subject bias is not an issue.

In Experiment 1, we examined the effects of noise, density, and corrugation frequency for the detection of single sine wave corrugated surfaces. In Experiment 4, we examined a subset of these conditions to determine whether similar patterns of performance would be obtained using a different paradigm with feedback.

\section{Method}

Subjects. The subjects were the 2 authors and 2 graduate students at the University of California, Riverside. The 2 graduate students were naive with regard to the purpose of the experiment and were paid for their participation. Acuity of at least 20/40 (Snellen Eye Chart) was required in the eye used in the experiment.

Design. Three independent variables were examined: spatial frequency of the corrugated surface $(0.57$ and 1.25 cycles/deg of visual angle), texture density (2.61 and $\left.7.2 \mathrm{dots} / \mathrm{deg}^{2}\right)$, and the standard deviation of velocity noise $(0,0.22,0.67$, or $1.12 \mathrm{deg} / \mathrm{sec})$.

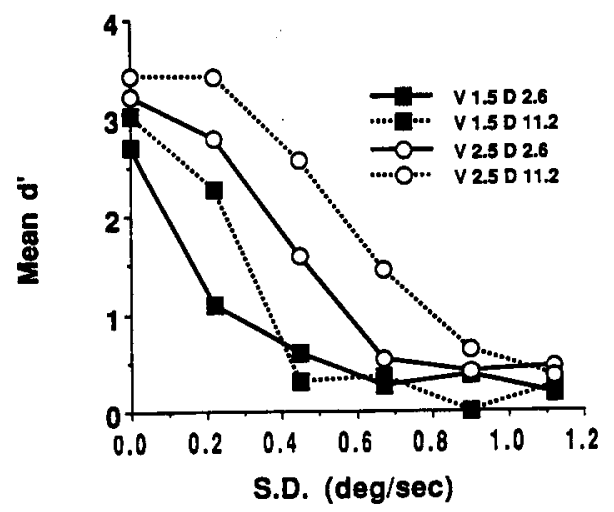

Figure 10. Mean $d^{\prime}$ as a function of density (D), amplitude (V), and velocity noise from Experiment 3 . 


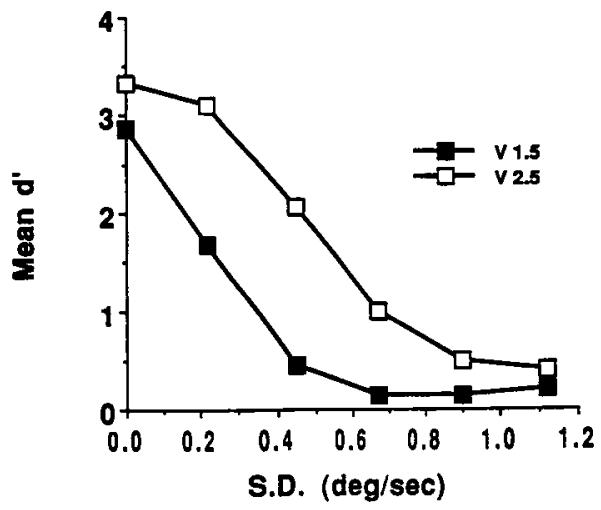

Figure 11. Mean $d^{\prime}$ as a function of amplitude $(V)$ and velocity noise from Experiment 3.

Stimuli and Apparatus. The stimuli and apparatus were identical to those used in the previous experiments.

Procedure. The procedure was the same as Experiments 1, 2, and 3, with the following exceptions. Each trial consisted of a surface and matched random display condition (same velocities randomized vertically) presented sequentially. The presentation order was randomized from trial to trial. Each subject viewed 25 trials of each corrugation frequency, density, and noise condition with the trials blocked by frequency and density. If the subject responded incorrectly to a trial, a tone was immediately presented for $1 \mathrm{sec}$ to inform the subject that the response was incorrect.

\section{Results and Discussion}

A $d^{\prime}$ was computed (the hit rate was the proportion of trials on which subjects correctly identified the surface display) for each subject for each surface frequency, velocity noise, and density level (Green \& Swets, 1966). A within-subjects three-way (velocity noise $X$ frequency $X$ density) ANOVA was conducted using the $d$ 's as the dependent variable. The main effect for velocity noise was significant $[F(3,9)=23.61, p<.01]$. Average $d$ 's for the $0,0.22,0.67$, and $1.12 \mathrm{deg} / \mathrm{sec}$ noise conditions were 2.17 , $1.85, .51$, and .37 , respectively. Post hoc comparisons indicated significant differences $(p<.05)$ between the 0 and $0.67,0$ and $1.12,0.22$ and 0.67 , and 0.22 and 1.12 noise conditions. The main effect of density was significant $[F(1,3)=12.85, p<.05]$, indicating greater detection performance for the $7.2 \mathrm{dots} / \mathrm{deg}^{2}$ condition (average $d^{\prime}$ of 2.4) than for the $2.61 \mathrm{dots} / \mathrm{deg}^{2}$ condition (average $d^{\prime}$ of .79). The main effect of corrugation frequency was significant $[F(1,3)=98.7, p<.01]$. Average $d$ 's for the 0.57 and 1.25 cycles/deg conditions were 1.88 and 0.57 , respectively.

An important issue is whether the use of feedback resulted in improved performance over the duration of the study. To assess this issue, we compared detection performance for the first 15 trials and last 15 trials for each surface condition for each subject. Separate $t$ tests were performed for Experiment 1, which did not use feedback, and Experiment 4, which did use feedback. The results indicated no significant differences between the first 15 and last 15 trials for either Experiment 1 or $4(p>.05)$.
Overall, the results using a $2 \mathrm{AFC}$ paradigm with feedback were similar to the results obtained in Experiment 1, with detection performance decreasing with an increase in noise, an increase in corrugation frequency, and a decrease in texture density.

\section{EXPERIMENT 5: CONTROL STUDY}

In Experiment 4, we examined the effects of noise, density, and corrugation frequency for the detection of single sine wave corrugated surfaces using a $2 \mathrm{AFC}$ paradigm with feedback. In the present experiment, we examined the importance of amplitude, noise, and corrugation frequency observed in Experiment 2 using a $2 \mathrm{AFC}$ paradigm with feedback.

\section{Method}

Subjects. The subjects were the 2 authors and 2 graduate students at the University of California, Riverside. The 2 graduate students were naive with regard to the purpose of the experiment and were paid for their participation. Acuity of at least 20/40 (Snellen Eye Chart) was required in the eye used in the experiment.

Design. Three independent variables were examined: depth range of the display (maximum to minimum velocity ratio of 1.5 or 2.5 ), corrugation frequency ( .58 or $.95 \mathrm{cycles} / \mathrm{deg})$, and the standard deviation of Gaussian noise $(0, .22, .67$, or $1.12 \mathrm{deg} / \mathrm{sec})$.

Stimuli and Apparatus. The stimuli and apparatus were identical to those used in the second experiment with the following exception. The texture density of the displays was $2.81 \mathrm{dots} / \mathrm{deg}^{2}$ for all displays.

Procedure. The procedure was the same as in Experiment 4.

\section{Results and Discussion}

A $d^{\prime}$ was computed (the hit rate was the proportion of trials on which subjects correctly identified the surface display) for each subject for each surface frequency, amplitude, velocity noise, and density level (Green \& Swets, 1966). A within-subjects three-way (frequency $\times$ velocity noise $\times$ amplitude) ANOVA was conducted using the $d$ 's as the dependent variable. The main effect for variance was significant $[F(3,9)=6.06, p<.025]$. Average $d$ 's for the $0,0.22,0.67$, and $1.12 \mathrm{deg} / \mathrm{sec}$ noise conditions were $3.55,2.96,2.23$, and 1.0, respectively. Post hoc com-

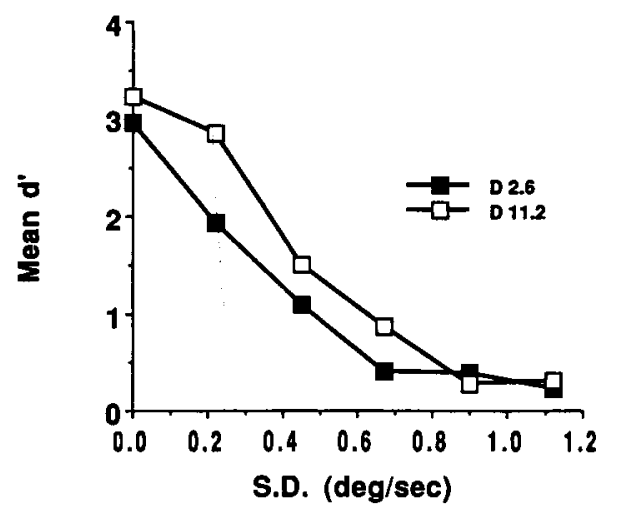

Figure 12. Mean $d^{\prime}$ as a function of density (D) and velocity noise from Experiment 3. 
parisons indicated significant differences $(p<.05)$ between the 0 and 1.12 , and 0.22 and 1.12 noise conditions. The main effect of amplitude was significant $[F(1,3)=$ $109.0, p<.01]$, indicating greater detection performance for the 1.5 velocity ratio (average $d^{\prime}$ of 1.74 ) than for the 2.5 velocity ratio condition (average $d^{\prime}$ of 3.2 ). The twoway interaction between variance and frequency was significant $[F(3,9)=5.72, p<.025]$.

Overall, the results using a $2 \mathrm{AFC}$ paradigm with feedback were similar to the results obtained in Experiment 2, with detection performance decreasing with an increase in noise, decreasing with an increase in amplitude, decreasing with an increase in corrugation frequency, and decreasing with a decrease in texture density.

\section{GENERAL DISCUSSION}

Previous studies (Andersen, 1996; Turner et al., 1995) have suggested that surface detection from optic flow (perspective projections) or structure from motion (orthographic projections) is based on smooth variations of the velocity field. The present study is the first systematic examination of the importance of smoothness of the velocity field for the detection of 3-D surfaces from optic flow. Subjects were presented with optic flow displays simulating 3-D corrugated surfaces. The smoothness of the velocity field defining the surfaces was varied by systematically adding velocity noise from a Gaussian distribution with $M=0$ and $S D=\sigma$ to the velocities of the display.

The first experiment assessed the effect of velocity noise in detecting corrugated surfaces of varying frequency and density. Detection performance decreased with an increase in velocity noise. The decrease in detection performance with an increase in velocity noise was greater for low- than for high-density conditions. In addition, the decrease in detection as a function of noise was greater for high- than for low-corrugation frequency surfaces.

Experiment 2 examined the effect of noise when corrugation frequency, amplitude, and texture density were varied. Detection performance also decreased with an increase in velocity noise. In addition, the decrease in detection performance was greater for high- than for lowamplitude surfaces.

The third experiment examined the importance of smoothness in detecting complex sinusoidal corrugated surfaces. Detection performance decreased with an increase in velocity noise in a manner similar to that found in Experiments 1 and 2. The effects of velocity noise on detection performance was greater for high- than for lowamplitude surfaces. In addition, the effects of noise on detection performance were greater for low- than for highdensity displays. These results, considered along with the results of Experiments 1 and 2, indicate that similar effects of velocity noise were obtained for the detection of simple (single sine wave) and complex (sums of sine waves) surfaces.

Overall, the results indicate that the visual system has a high tolerance for noise in detecting 3-D corrugated surfaces from optic flow. Performance decreased systematically and in a graceful manner with increased velocity noise for each experiment. The effects of noise on surface detection varied as a function of the frequency, amplitude, and density of the display. Thus, the results of the present experiments are not consistent with a critical value of noise in which the detection of surfaces with noise equal to or greater than such a value is at chance.

An analysis based on smooth variations of velocity field has been proposed for the detection of 3-D surfaces from optic flow (Andersen, 1996) and structure from motion (Turner et al., 1995). ${ }^{1}$ Andersen (1996) proposed an analysis based on the summed value of the two shear components (Koenderink, 1986) of deformation determined by two shear detectors (Figure 13). The analysis of smoothness using the two shear components of the velocity field was derived by calculating an average output of the four receptive fields of the two shear components (Figure 13). Analyses of the shear components of deformation using single sine and complex sine wave corrugated surfaces yielded $d^{\prime}$ values that were significantly correlated with the mean $d^{\prime}$ values derived from human observers.

Can this analysis also account for the performance observed in the present study when the smoothness of the flow field was varied? In order to examine this possibility, simulations were conducted in which a lattice of two layers of shear detectors - one layer for each type of shear detector $\left(\Sigma_{d 1}\right.$ and $\left.\Sigma_{d 2}\right)$-was used to analyze the displays examined in the experiments (Figure 13). Each layer of the lattice consisted of a $25 \times 25$ array of sampling regions (each region sampling velocities in a $1.2^{\circ}$ diameter area of the display, with a $66 \%$ spatial overlap of adjacent regions) with both types of detectors sampling velocities in each region. For each of the four receptive fields of each detector, a scalar product $A \cdot B$ (where $A$ is the average velocity of the dots within a receptive field divided by the maximum velocity of the display and $B$ is a unit vector) was calculated, producing values ranging between -1 and 1 . The output of either detector $\Sigma_{d 1}$ or $\Sigma_{d 2}$ of the lattice was simply the sum of the four receptive fields of that detector.

A summed absolute value was then calculated between the two detectors $\left(\Sigma_{d 1}\right.$ and $\left.\Sigma_{d 2}\right)$ sampling the same image region, and these were averaged across all sample regions in the image. Since the average summed absolute value for any given display will vary according to the sample of velocities in the display, Monte Carlo simulations were conducted to derive distributions based on the average $\sum_{d(\text { sum })}$ for surface and random conditions. A $d^{\prime}$ statistic (surface distribution mean minus random distribution mean divided by the larger standard deviation of the two distributions) was calculated for each condition in the experiments on the basis of the computed means and standard deviations of the distributions produced by the Monte Carlo simulations (Figure 14).

The simulations were based on two frames from the displays, with 30 samples of each display condition used to derive distributions for surface and random conditions. 


\section{Lattice of Shear Detectors}

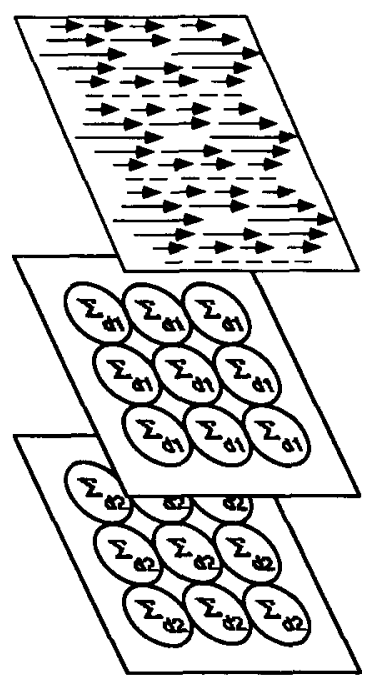

\section{Optic Flow Field}

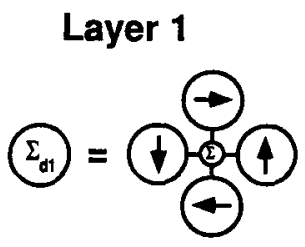

Layer 2

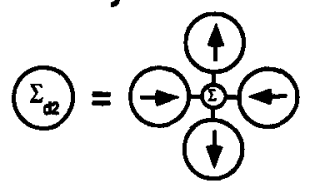

Figure 13. The two-layer lattice of shear detectors used to analyze the deformation component of optic flow. $\Sigma_{d 1}$ and $\Sigma_{d 2}$ refer to the two types of shear detectors proposed by Koenderink and van Doorn (1977). The optic flow field was filtered by the two layers of the lattice, with an absolute sum value of $\Sigma_{d 1}+\Sigma_{d 2}$ derived for each pair of detectors at each location of the image. See text for a detailed description of this analysis.

The velocity field generated from the two frames assumed that correspondence was solved. The analysis focused on the results from Experiments 2 and 3 since these experiments examined density, corrugation frequency, and corrugation amplitude - the variables found to be important for the detection of 3-D corrugated surfaces. In addition, Experiments 2 and 3 examined simple and complex corrugated surfaces, respectively.

The overall pattern of results for displays examined in these experiments was similar to the pattern of results obtained for human observers. As shown in Figure 5, the predicted $d^{\prime}$ for Experiment 2 increased with an increase in the texture density, an increase in corrugation amplitude, and with a decrease in corrugation frequency. The direction of these results was the same as that obtained with human observers in Experiment 2. To assess the goodness-of-fit between the model and human performance, a correlation was computed across the 72 surface and noise display conditions between the predicted $d^{\prime}$ and the average $d^{\prime}$ of the 4 observers. The correlation $(r=.93)$ was significant $[t(70)=14.2, p<.01]$ and accounted for $86 \%$ of the variance in the condition means. Correlations for specific surface displays, across variations in noise, ranged from .76 to .97 .

The predicted $d$ 's for Experiment 3 are shown in Figure 8 . The pattern of results for predicted $d^{\prime}$ was again similar to the results obtained with human observers. The predicted $d^{\prime}$ decreased with an increase in noise and with a decrease in surface amplitude. The correlation between predicted ( 30 surface and noise display condi-

tions) and average $d^{\prime}$ (of the 4 observers) was $r=.97$; this was significant $[t(46)=22.4, p<.01]$ and accounted for $94 \%$ of the variance in the condition means. Correlations for specific surface displays, across variations in noise, ranged from .93 to .98 . These results provide support for the proposal that the detection of 3-D corrugated surfaces was consistent with an analysis based on the smoothness of the velocity field using the two shear components of deformation of the optic flow field.

The results of the present study, considered with the results of studies examining velocity distributions (Atchley \& Andersen, 1995) and overlapping velocity fields (Andersen, 1989; Andersen \& Wuestefeld, 1993), suggest that the analysis of velocity fields involves a twostage process. In the first stage, the visual system segregates the velocity field into component distributions of velocity. In the second stage, a deformation analysis is performed on velocity distributions for the detection and recovery of 3-D surfaces. The experiments in the present study, as well as our previous research on velocity distributions, have exclusively examined perspective translations. An important goal for future research is to assess the generality of this approach by examining transformations involving orthographic rotation.

In summary, the present results indicate the tolerance of the visual system in detecting 3-D surfaces from optic flow when smoothness of the velocity field was varied by adding noise velocities to the display. Our results indicate that human observers were quite good at detecting corrugated surfaces that varied in frequency, amplitude, and complexity when velocity noise was present in the flow field. The results indicate that detection performance degraded gracefully with an increase in velocity noise. The effects of velocity noise on surface detection varied according to the corrugation frequency, amplitude, and density of the display, indicating that there is no specific noise velocity value for which performance, across all conditions, drops to chance. An analysis of the two local shear components of the displays provided a reasonably

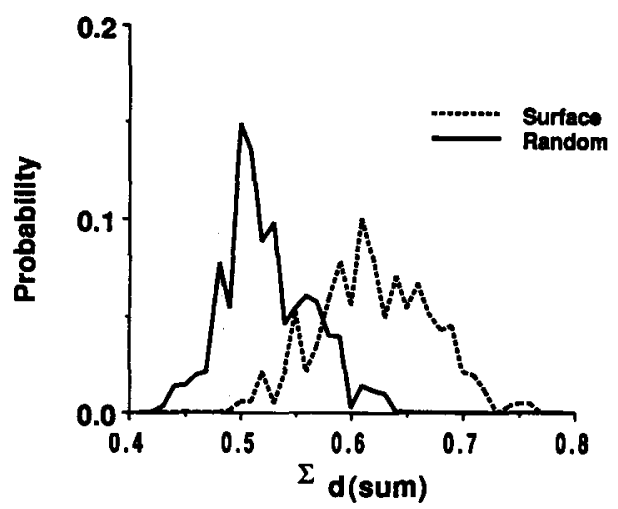

Figure 14. Relative frequency distributions of average $\mathbf{\Sigma}_{d(\text { sum })}$. The two distributions are for a single surface and random display pair (frequency of 0.58 cycles/deg; velocity ratio of 2.5 , density of $2.6 \mathrm{dots} / \mathrm{deg}^{2}$, standard deviation of Gaussian noise of 0.67 ) from Experiment 2. The distributions are based on 900 Monte Carlo runs. 
good fit to the performance of human observers for detecting surfaces that varied in frequency, amplitude, and density. The results of the present study thus provide further support for the importance of the two shear components of deformation for the detection of 3-D surfaces from optic flow.

\section{REFERENCES}

ANDERSEN, G. J. (1989). Perception of three-dimensional structure from optic flow without locally smooth velocity. Journal of Experimental Psychology: Human Perception \& Performance, 15, 49-55.

ANDERSEN, G. J. (1996). Detection of smooth three-dimensional surfaces from optic flow. Journal of Experimental Psychology: Human Perception \& Performance, 22, 945-957.

ANDERSEN, G. J., \& Wuestefeld, A. P. (1993). Detection of threedimensional surfaces from optic flow: The effects of noise. Perception \& Psychophysics, 54, 321-333.

Atchley, P. A., \& ANDersen, G. J. (1995). Discrimination of speed distributions: Sensitivity to statistical properties. Vision Research, 35, 3131-3144.

Braunstein, M. L., \& Andersen, G. J. (1981). Velocity gradients and relative depth perception. Perception \& Psychophysics, 29, 145-155.

Braunstein, M. L., Hoffman, D. D., \& Pollick, F. E. (1990). Discriminating rigid from nonrigid motion: Minimum points and views. Perception \& Psychophysics, 47, 205-214.

Braunstein, M. L., \& Payne, J. W. (1969). Perspective and form ratio as determinants of relative slant judgments. Journal of Experimental Psychology, 81, 584-590.

Braunstein, M. L., \& Titrle, J. S. (1988). The observer-relative velocity field as the basis for effective motion parallax. Journal of Experimental Psychology: Human Perception \& Performance, 15, 582. 590.

DE BRUYN, B., \& OrBan, G. A. (1990). The importance of velocity gradients in the perception of three-dimensional rigidity. Perception, 19, 21-27.

De Bruyn, B., \& Orban, G. A. (1993). Segregation of spatially superimposed optic flow components. Journal of Experimental Psychology: Human Perception \& Performance, 19, 1014-1027.

Droulez, J., \& CorNilleau-PÉrÈs, V. (1990). Visual perception of surface curvature: The spin variation and its physiological implications. Biological Cybernetics, 62, 211-224.

GiBson, J. J. (1966). The senses considered as perceptual systems. Boston: Houghton Mifflin.

GrEen, D. A., \& SwETS, J. A. (1966). Signal detection theory and psychophysics. New York: Wiley.

Helmholtz, H. L. F. voN (1962). Treatise on physiological optics (Vol. 3, J. P. C. Southall, Ed. \& Trans.). New York: Dover. (Original work published in German, 1867, and in English, 1925)

KoENDERINK, J. J. (1986). Optic flow. Vision Research, 26, 161-180.

KoENDERINK, J. J., \& VAN DOORN, A. J. (1977). How an ambulant observer can construct a model of the environment from the geometric structure of the visual inflow. In G. Hauske \& E. Butendant (Eds.), Kibernetic (pp. 235-252). Munich: Oldenbourg.

Longuet-Higgins, H. C., \& Prazdny, K. (1980). The interpretation of a moving retinal image. Proceedings of the Royal Society of London: Series $B, 208,385-397$.

NoRman, J. F., \& LAPPIN, J. S. (1992). The detection of surface curvatures defined by optical motion. Perception \& Psychophysics, 51, 386-396.

Rogers, B., \& GRAHAM, M. (1979). Motion parallax as an independent cue for depth perception. Perception, 8, 125-134.

ToDD, J. T. (1984). The perception of three-dimensional structure from rigid and nonrigid motion. Perception \& Psychophysics, 36, 97-103.

TURNER, J., \& BRAUNSTEIN, M. L. (1994). Detection of a gap in transparent motion displays. Investigative Ophthalmology \& Visual Science, 35 ( 3 , Suppl.), 2157.

Turner, J., Braunstein, M. L., \& Andersen, G. J. (1995). Detection of surfaces in structure from motion. Journal of Experimental Psychology: Human Perception \& Performance, 21, 809-821.

VERRI, A., Girosi, F., \& TorRe, V. (1990). Differential techniques for optical flow. Journal of the Optical Society of America A, 7, 912-922.

\section{NOTE}

1. The Turner et al. (1995) analysis is appropriate for displays with small numbers of points and thus was not examined in the present study.

(Manuscript received September 12, 1995; revision accepted for publication May 2, 1996.) 